RASĀYAN J. Chem.

Vol. 13 | No. 4 |2552-2558| October - December | 2020 ISSN: 0974-1496 | e-ISSN: 0976-0083 | CODEN: RJCABP

\title{
ISOLATION AND CHARACTERIZATION OF STIGMASTEROL AND $\beta$-SITOSTEROL FROM WOOD BARK EXTRACT OF Baccaurea macrocarpa Miq. Mull. Arg
}

\author{
Erwin $^{1, *}$, W.R. Pusparohmana ${ }^{1}$, R.D. Safitry ${ }^{1}$, E. Marliana ${ }^{1}$, Usman $^{2}$ \\ and I.W. Kusuma ${ }^{3}$ \\ ${ }^{1}$ Department of Chemistry, Mathematics and Natural Sciences Faculty, Mulawarman University, \\ Samarinda-75119, (East Kalimantan) Indonesia \\ ${ }^{2}$ Study Program of Chemical Education, Faculty of Teacher Trainer and Education/ \\ Mulawarman University, Samarinda-75119, (East Kalimantan) Indonesia \\ ${ }^{3}$ Faculty of Forestry, Mulawarman University, Samarinda-75119, (East Kalimantan) Indonesia \\ *E-mail: winulica@yahoo.co.id
}

\begin{abstract}
Baccaurea macrocarpa Miq. Mull. Arg. (known locally as Tampoi) is one of the edible fruit plants found in the forests of Borneo. The crude extract of wood bark of Tampoi was partitioned with $n$-hexane and ethyl acetate successively to yield respectively soluble fractions to biological activity assay. The toxicity was measured by the brine shrimp lethality test method, and the antioxidant activity was carried out by the DPPH radical scavenging method. While the isolation and purification were carried out using flash column chromatography. The results of the biological assay showed that the ethyl acetate fraction was the most active in the antioxidant activity test, with $\mathrm{IC}_{50}$ values $35.56 \mu \mathrm{g} / \mathrm{ml}$, and none of the fractions is toxic. Isolation and purification of the ethyl acetate fraction gave white crystalline powder with a melting point $129-130{ }^{\circ} \mathrm{C}$. Characterization of the compound based on FT-IR, ${ }^{1} \mathrm{H}$, ${ }^{13} \mathrm{C}-\mathrm{NMR}, \mathrm{NMR} 2 \mathrm{D}$ spectra and comparison to that of the published NMR data suggested that the compound (1) was a mixture of stigmasterol and $\beta$-sitosterol.
\end{abstract}

Keywords: Baccaurea macrocarpa, Toxicity, Characterization, Antioxidants, Stigmasterol, $\beta$-sitosterol.

(C) RASĀYAN. All rights reserved

\section{INTRODUCTION}

East Kalimantan is one of the provinces in Indonesia having tropical rain forests. Diversity of tropical plants contained in it one of which is the genus of Baccaurea. Generally, Baccaurea plants have edible fruits, and some of them are traditionally used as medicine. Baccaurea is a reasonably large genus; around 38 species of Baccaurea are recognized. The distribution of this plant genus includes India, Burma, Malaysia, Borneo, Sumatra, the Philippines, Thailand, Papua New Guinea, Sulawesi (Talaud Island), Bali, and the Pacific islands ${ }^{1}$. Utilization of Baccaurea as an alternative medicine such as to treat arthritis, abdominal pain, eye pain, abscesses, constipation, facilitates urination and menstruation. Previous research results also showed that Baccaurea has the potential as an anticancer, antidiabetic, antioxidant, anti-inflammatory, antimicrobial, and antitrypanosomal agents ${ }^{1-4}$. However, based on the literature search, no one has reported secondary metabolites isolated from Tampoi. The previous studies have shown crude extracts of Tampoi wood bark is very active as an antioxidant ${ }^{5}$. This study is a continuation of research aimed to characterize, identify and determine the toxicity against Artemia salina $\mathrm{L}$ and antioxidant activity against DPPH radical scavenging of the compound obtained from the Baccaurea macrocarpa (Miq.) Mull. Arg (Tampoi) wood bark extract.

\section{EXPERIMENTAL}

\section{Material}

The sample of this research was the wood bark of B. macrocarpa (Miq.) Mull. Arg. (Tampoi) Collected from Kedang Ipil Village, Kota Bangun, Kutai Kartanegara. Methanol, ethyl acetate, and $n$-Hexane were

Rasayan J. Chem., 13(4), 2552-2558(2020)

http://dx.doi.org/10.31788/ RJC.2020.1345652

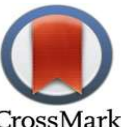


RASĀYAN J. Chem.

Vol. 13 | No. 4 |2552-2558| October - December | 2020

used in the extraction, chromatography, and purification section. TLC Silica Gel 60 F254 (1.05554.0001) and Kieselgel 60 (1.07734.1000) were used for TLC analysis and flash column chromatography, respectively.

\section{Instrumentation}

FTIR spectrum was measured using FTIR Prestige 21 (Shimadzu Corp, Japan. Whereas the ${ }^{1} \mathrm{H}$ - and ${ }^{13} \mathrm{C}$ NMR spectrum including NMR-2D was measured using a $500 \mathrm{MHz}$ Agilent DD2 NMR Spectrometer, which operates at frequencies of $500 \mathrm{MHz}\left({ }^{1} \mathrm{H}\right)$ and $125 \mathrm{MHz}\left({ }^{13} \mathrm{C}\right)$.

\section{General Procedure}

\section{Extraction, Isolation, and Purification}

A total of 180 grams of Tampoi wood bark extract was re-dissolved into methanol then partitioned with $n$-hexane and ethyl acetate successively. After the solvent removal using a rotary evaporator, the fractions of $n$-hexane ( $20 \mathrm{~g})$, ethyl acetate $(40 \mathrm{~g})$, and methanol ( $80 \mathrm{grams})$ were obtained. The ethyl acetate fraction (40 grams) was further fractionated using vacuum column chromatography using ethyl acetate: $n$ hexane mixture eluent $(5: 95-100: 0)$ and 37 vials were obtained. The fractions were combined into five fractions, E1 (346.7 mg), E2 (579.4 mg), E3 $(276.3 \mathrm{mg}), \mathrm{E} 4(353.5 \mathrm{mg})$, and E5 $(3245.5 \mathrm{mg})$ based on TLC spot profile. E2 fraction $(579.4 \mathrm{mg})$ was isolated by flash column chromatography using a mixture of eluent ethyl acetate: $n$-hexane (1: 9). Fraction E2 (579 $\mathrm{mg})$ was isolated by flash column chromatography using a mixture eluent ethyl acetate: $n$-hexane (1: 9) to give 5 main fractions, namely E2.1 (31 mg), E2.2 (68 mg), E2.3 (67.3 mg), E2.4 (104 mg) and E2.5 $(54.3 \mathrm{mg})$. Thirty $\mathrm{mg}$ of white crystalline powder was obtained after recrystallization of E2.2.

The purity test using thin-layer chromatography analysis on three eluent variations, showing the formation of a single spot with an Rf value of 0.27 (chloroforms: $n$-hexane $=4: 6$ ), 0.33 (ethyl acetate: $n$ hexane $=1: 9)$, and $0.38(100 \%$ chloroforms $)$. Melting point measurement displayed that the compound (1) had m.p. $129-130{ }^{\circ} \mathrm{C}$.

\section{Toxicity Tests}

Toxicity tests were performed using the brine shrimp lethality test method against Artemia salina L. The samples were dissolved into $500,250,125,62.5,31.25,15.63$, and $7.81 \mathrm{ppm}$. Each sample solution is inserted between 8-15 shrimp larvae. In the same way, blanks are made without being sampled. Both samples and blanks were repeated three times. ${ }^{5-7}$

\section{Antioxidant Activity Test}

The antioxidant test was performed using the DPPH free radical scavenging method refers to the previous research method. Inhibition of the sample against the DPPH free radical was calculated according to the formula: Inhibition (\%) $=[(\mathrm{A}-\mathrm{A} 1) / \mathrm{A}] \mathrm{X} 100$. Meanwhile, the determination of $\mathrm{LC}_{50}$ was carried out using linear regression on concentration vs inhibition (\%), where, $\mathrm{A}=$ absorbance of blank and $\mathrm{A} 1=$ absorbance of the sample..$^{5,8-15}$

\section{Steroid Test of compound (1)}

A few mg of compound (1) was put into a test tube, then a few drops of Liebermann-Burchard reagent were added (glacial acetic acid + concentrated $\mathrm{H}_{2} \mathrm{SO}_{4}$ ). The formation of green indicates compound 1 is a steroid. ${ }^{5,8,16}$

\section{Spectroscopic Data}

Spectroscopic data measurements of compound (1) were comprised of FT-IR, ${ }^{1} \mathrm{H}-\mathrm{NMR},{ }^{13} \mathrm{C}-\mathrm{NMR}$, and NMR-2D. IR spectrum data were recorded using a Shimadzu FTIR Prestige 21 (Shimadzu, Japan). NMR spectra were recorded using the $500 \mathrm{MHz}$ NMR Agilent with DD2 console system operating at frequencies of $500 \mathrm{MHz}\left({ }^{1} \mathrm{H}\right)$ and $125 \mathrm{MHz}\left({ }^{13} \mathrm{C}\right)$ using $\mathrm{CDCl}_{3}$ as a solvent in the ITB Chemistry Department. Compound (1) was obtained as a white powder with a melting point of $129-130{ }^{\circ} \mathrm{C}$. FT-IR spectrum data showed the absorption peaks at $3427.51 \mathrm{~cm}^{-1}(\mathrm{OH}), 3050.00 \mathrm{~cm}^{-1}\left(\mathrm{CH}\right.$ alkene), $2866.22 \mathrm{~cm}^{-}$ ${ }^{1}, 2935.66 \mathrm{~cm}^{-1}$, and $1463.97 \mathrm{~cm}^{-1}$ (CH aliphatic), $1658.78 \mathrm{~cm}^{-1}(\mathrm{C}=\mathrm{C}), 1134.14 \mathrm{~cm}^{-1}(\mathrm{CO})$. The ${ }^{1} \mathrm{H}-$ and 
RASĀYAN J. Chem.

Vol. 13 | No. 4 |2552-2558| October - December | 2020

${ }^{13} \mathrm{C}$-NMR spectra of compound (1) were the entirety of the stigmasterol and $\beta$-sitosterol data as listed in Table-3.

\section{RESULTS AND DISCUSSION}

Partitions of 180 grams of crude extract of Tampoi wood bark yielded $n$-hexane, ethyl acetate, and methanol fractions of 8,20 , and 40 grams, respectively. The results of toxicity tests against larval of Artemia salina showed that all fractions were not toxic $\left(\mathrm{LC}_{50}>1000 \mathrm{ppm}\right)^{6}$, as presented in Table-1.

Table-1: $\mathrm{LC}_{50}$ Value of Fractions and Compound (1) (the concentrations, total larvae, and dead larvae were the averages of three replicates).

\begin{tabular}{|c|c|c|c|c|c|c|c|c|}
\hline Sample & Concentration & $\begin{array}{c}\mathrm{Log} \\
\text { Concentration }\end{array}$ & $\begin{array}{c}\text { Total } \\
\text { Larvae }\end{array}$ & $\begin{array}{c}\text { Dead } \\
\text { Larvae }\end{array}$ & $\begin{array}{c}\% \\
\text { Mortality }\end{array}$ & Probit & $\begin{array}{c}\text { Linear } \\
\text { Regressio } \\
\mathrm{n}\end{array}$ & $\begin{array}{l}\mathrm{LC}_{50} \\
(\mathrm{ppm})\end{array}$ \\
\hline \multirow{7}{*}{$\begin{array}{l}n \text {-hexane } \\
\text { fraction }\end{array}$} & 500 & 2.6989 & 9.7 & 4.7 & 48.4 & 4.95 & \multirow{7}{*}{$\begin{array}{l}y= \\
0.3773 x+ \\
3.591\end{array}$} & \multirow[t]{7}{*}{5425.36} \\
\hline & 250 & 2.3979 & 11 & 3 & 27.2 & 4.39 & & \\
\hline & 125 & 2.0969 & 9.7 & 2.3 & 23.7 & 4.26 & & \\
\hline & 62.5 & 1.7959 & 10.3 & 1.7 & 16.5 & 4.01 & & \\
\hline & 31.25 & 1.4948 & 10.7 & 2.3 & 21.5 & 4.19 & & \\
\hline & 15.63 & 1.1938 & 10 & 1 & 10 & 3.72 & & \\
\hline & 7.81 & 0.8928 & 10.3 & 2.7 & 26.2 & 4.36 & & \\
\hline \multirow{7}{*}{$\begin{array}{l}\text { Ethyl } \\
\text { acetate } \\
\text { fraction }\end{array}$} & 500 & 2.6989 & 10.3 & 7.7 & 74.7 & 5.64 & \multirow{7}{*}{$\begin{array}{l}y= \\
0.0819 x+ \\
4.6659\end{array}$} & \multirow[t]{7}{*}{12005.08} \\
\hline & 250 & 2.3979 & 8.3 & 2.3 & 27.7 & 4.39 & & \\
\hline & 125 & 2.0969 & 9.7 & 3 & 30.9 & 4.48 & & \\
\hline & 62.5 & 1.7959 & 10.7 & 4 & 37.3 & 4.67 & & \\
\hline & 31.25 & 1.4948 & 9.3 & 3.3 & 35.5 & 4.61 & & \\
\hline & 15.63 & 1.1938 & 10 & 4.3 & 43 & 4.82 & & \\
\hline & 7.81 & 0.8928 & 9.3 & 5 & 53.8 & 5.08 & & \\
\hline \multirow{7}{*}{$\begin{array}{l}\text { Methanol } \\
\text { fraction }\end{array}$} & 500 & 2.6989 & 8.3 & 3.3 & 39.7 & 4.72 & \multirow{7}{*}{$\begin{array}{l}y= \\
0.2598 x+ \\
3.8505\end{array}$} & \multirow[t]{7}{*}{26580.15} \\
\hline & 250 & 2.3979 & 10.7 & 2 & 18.7 & 4.08 & & \\
\hline & 125 & 2.0969 & 10.3 & 3 & 29.1 & 4.45 & & \\
\hline & 62.5 & 1.7959 & 10.3 & 3.7 & 35.9 & 4.61 & & \\
\hline & 31.25 & 1.4948 & 10.7 & 2.3 & 21.5 & 4.19 & & \\
\hline & 15.63 & 1.1938 & 11.7 & 2.3 & 19.6 & 4.12 & & \\
\hline & 7.81 & 0.8928 & 10 & 1.7 & 17 & 4.05 & & \\
\hline \multirow{7}{*}{$\begin{array}{l}\text { Compoun } \\
\text { d (1) }\end{array}$} & 500 & 2.6989 & 10 & 4.7 & 47 & 4.92 & \multirow{7}{*}{$\begin{array}{l}Y= \\
-0.0261 \mathrm{x} \\
+5.114\end{array}$} & \multirow[t]{7}{*}{23324.70} \\
\hline & 250 & 2.3979 & 10 & 6 & 60 & 5.25 & & \\
\hline & 125 & 2.0969 & 10 & 4.7 & 47 & 4.92 & & \\
\hline & 62.5 & 1.7959 & 10 & 5.7 & 57 & 5.18 & & \\
\hline & 31.25 & 1.4948 & 10 & 5 & 50 & 5.00 & & \\
\hline & 15.63 & 1.1938 & 10,3 & 6 & 58,3 & 5.20 & & \\
\hline & 7.81 & 0.8928 & 10 & 5 & 50 & 5.00 & & \\
\hline
\end{tabular}

While the antioxidant test results using DPPH free radical method showed that the ethyl acetate fraction was the most active, as shown in Table-2.

Isolation and purification of ethyl acetate fraction gave compound (1) as a white powder with a melting point of $129-130{ }^{\circ} \mathrm{C}$. FT-IR spectrum data showed that the absorption of $3427.51 \mathrm{~cm}^{-1}$ (hydroxyl groups) was supported by $1134.14 \mathrm{~cm}^{-1}$ (Secondary alcohol, C-O stretch). Absorption of stretching at 2935.66 and $2866.22 \mathrm{~cm}^{-1}$ indicated the presence of $\mathrm{CH}$ aliphatic supported by the absorption at $1463.97 \mathrm{~cm}^{-1}$ (for cyclic $\mathrm{CH}_{2}$ ). Other absorption at $3050.00 \mathrm{~cm}^{-1}$ due to $=\mathrm{CH}$ structure and it was endorsed by $1658.78 \mathrm{~cm}^{-1}$ $(\mathrm{C}=\mathrm{C}$ stretch $)$. The qualitative test results against Liebermann-Burchard reagents formed in green indicated the compound (1) has a steroid nucleus.

${ }^{1} \mathrm{H}-\mathrm{NMR}$ spectrum data showed the presence of a signal at $3.52(\mathrm{~m}, 1 \mathrm{H})$ for $\mathrm{H}-3$ and at $5.36(\mathrm{t}, 1 \mathrm{H})$ for H6. Two singlet signals 0.85 (s) and 0.10 (s) for $-\mathrm{CH}_{3}$ at $\mathrm{H}-18$ and $\mathrm{H}-19$, respectively. Two methyl doublet at $1.03(J=7.2 \mathrm{~Hz})(\mathrm{H}-21)$ and $1.02(\mathrm{~d}, J=13 \mathrm{~Hz})$ for stigmasterol $(1) / 0.83(J=11 \mathrm{~Hz})(\mathrm{H}-26)$ for $\beta$ - 
RASĀYAN J. Chem.

Vol. 13 | No. 4 |2552-2558| October - December | 2020

sitosterol (2), and one broad singlet at 0.84 (br s) (H-27). The presence of signals at 5.00, (dd, $J=1.73 \mathrm{~Hz}$ and $1.72 \mathrm{~Hz}$ ) and $5.15(\mathrm{dd}, J=1.75$ and 1.73$)$ are $\mathrm{H}-22$ and $\mathrm{H}-23$, respectively for Stigmasterol (1).

${ }^{13} \mathrm{C}$-NMR Spectrum data shows there were 50 signals overall. The signals at 140.87 (C5), 121.84 (C6), and 140.87 (C5), 121.85 (C6) were carbon double bonds for Stigmasterol and $\beta$-sitosterol, respectively. The signal at 71.93 was one carbon oxymetin $\mathrm{C}-\mathrm{sp}^{3}$ for $\mathrm{C} 3$. The presence of carbon double bonds was shown in signals at $8.46(\mathrm{C} 22)$ and 129.39 (C23) for stigmasterol (1). Stigmasterol and $\beta$-sitosterol are two types of steroids that have similar molecular formulas that differ only at C-22 and C-23. Based on NMR data, including NMR-2D and supported by literature data, compound (1) is a mixture of Stigmasterol and $\beta$-sitosterol. Stigmasterol and $\beta$-sitosterol, two plant sterols that are difficult to separate. Both of these compounds have almost the same polarity so that they are often obtained in mixed form ${ }^{17-20}$. The results of antioxidant tests of compounds (1) against free radical DPPH showed low antioxidant activity with an $\mathrm{LC}_{50}$ value of $74.33 \mathrm{ppm}$. The results of the toxicity test for compound (1) against Artemia salina larvae showed no toxicity with $\mathrm{LC}_{50}$ values above $1000 \mathrm{ppm}^{6}$.

Table-2. Antioxidant Activity of Fractions and Compound (1). (The concentrations and absorbances were the averages of three replicates)

\begin{tabular}{|c|c|c|c|c|c|c|}
\hline \multirow[t]{2}{*}{ Sample } & \multirow{2}{*}{$\begin{array}{c}\text { Concentration } \\
(\mathrm{ppm})\end{array}$} & \multicolumn{2}{|c|}{ Absorbance } & \multirow[t]{2}{*}{$\%$ Inhibition } & \multirow{2}{*}{$\begin{array}{c}\text { Linear } \\
\text { Regression }\end{array}$} & \multirow{2}{*}{$\begin{array}{c}\mathrm{IC}_{50} \\
(\mathrm{ppm})\end{array}$} \\
\hline & & Sample & Blank & & & \\
\hline \multirow{4}{*}{$\begin{array}{l}n \text {-hexane } \\
\text { fraction }\end{array}$} & 20 & 0.186 & \multirow{4}{*}{0.265} & 29.68 & \multirow{4}{*}{$\begin{aligned} \mathrm{Y} & =0.6358 \mathrm{x} \\
& +18.05\end{aligned}$} & \multirow{4}{*}{50.25} \\
\hline & 40 & 0.147 & & 44.52 & & \\
\hline & 60 & 0.113 & & 57.35 & & \\
\hline & 80 & 0.085 & & 67.80 & & \\
\hline \multirow{4}{*}{$\begin{array}{l}\text { Ethyl acetate } \\
\text { fraction }\end{array}$} & 20 & 0.153 & \multirow{4}{*}{0.265} & 42.26 & \multirow{4}{*}{$\begin{array}{c}\mathrm{Y}=0.6164 \mathrm{x}+ \\
29.371\end{array}$} & \multirow{4}{*}{33.47} \\
\hline & 40 & 0.124 & & 53.08 & & \\
\hline & 60 & 0.089 & & 66.54 & & \\
\hline & 80 & 0.056 & & 78.86 & & \\
\hline \multirow{4}{*}{$\begin{array}{l}\text { Methanol } \\
\text { fraction }\end{array}$} & 20 & 0.211 & \multirow{4}{*}{0.265} & 20.38 & \multirow{4}{*}{$\begin{array}{c}\mathrm{Y}=0.3748 \mathrm{x}+ \\
12.516\end{array}$} & \multirow{4}{*}{100.01} \\
\hline & 40 & 0.194 & & 26.92 & & \\
\hline & 60 & 0.172 & & 35.09 & & \\
\hline & 80 & 0.152 & & 42.64 & & \\
\hline \multirow{4}{*}{$\begin{array}{l}\text { Ascorbic } \\
\text { acid }\end{array}$} & 2 & 0.220 & \multirow{4}{*}{0.265} & 16.85 & \multirow{4}{*}{$\begin{array}{c}\mathrm{y}= \\
=9.5283 \mathrm{x}- \\
1.4465\end{array}$} & \multirow{4}{*}{5.40} \\
\hline & 4 & 0.167 & & 36.98 & & \\
\hline & 6 & 0.113 & & 57.36 & & \\
\hline & 8 & 0.070 & & 73.58 & & \\
\hline \multirow{4}{*}{$\begin{array}{c}\text { Compound } \\
\text { (1) }\end{array}$} & 20 & 0.157 & \multirow[t]{4}{*}{0.177} & 11.30 & \multirow{4}{*}{$\begin{aligned} \mathrm{y}= & 0.7043 \mathrm{x}- \\
& 2.354\end{aligned}$} & \multirow[t]{4}{*}{74.33} \\
\hline & 40 & 0.131 & & 25.80 & & \\
\hline & 60 & 0.104 & & 41.24 & & \\
\hline & 80 & 0.083 & & 53.11 & & \\
\hline
\end{tabular}
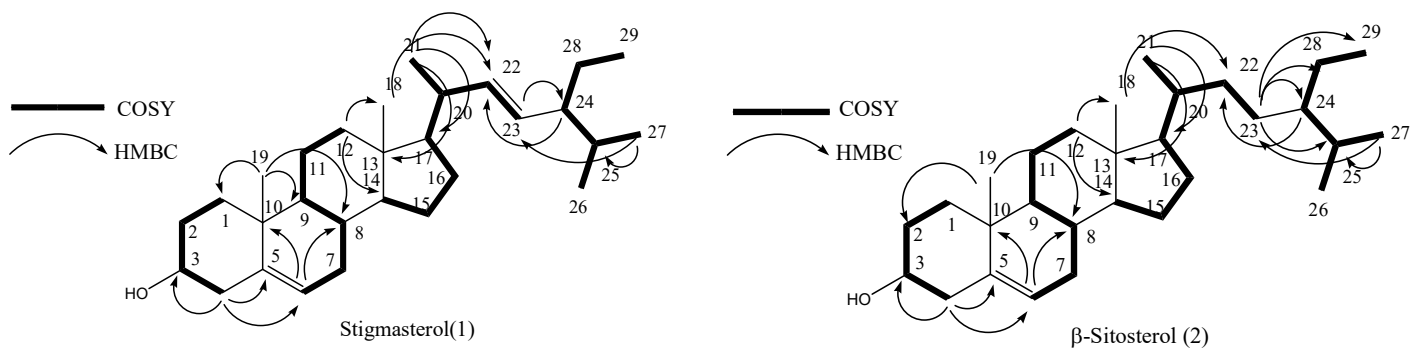

Fig.-1: Chemical Structure of Stigmasterol (1) and $\beta$-Sitosterol (2) 
RASĀYAN J. Chem.

Vol. 13 | No. 4 |2552-2558| October - December | 2020

Table-3: ${ }^{1} \mathrm{H}$ and ${ }^{13} \mathrm{C}-\mathrm{NMR}$ Spectrum Data for Stigmasterol (1) and $\beta$-sitosterol (2)

\begin{tabular}{|c|c|c|c|c|c|c|c|c|c|c|}
\hline \multirow[t]{3}{*}{ No } & \multicolumn{5}{|c|}{ Stigmasterol (1) } & \multicolumn{5}{|c|}{$\beta$-Sitosterol (2) } \\
\hline & \multicolumn{3}{|c|}{ Experimental } & \multicolumn{2}{|c|}{ Literature ${ }^{18}$} & \multicolumn{3}{|c|}{ Experimental } & \multicolumn{2}{|c|}{ Literature $^{18}$} \\
\hline & ${ }^{1} \mathrm{H}-\mathrm{NMR}$ & $\begin{array}{l}{ }^{13} \mathrm{C}- \\
\mathrm{NMR}\end{array}$ & $\begin{array}{c}\text { HMBC } \\
\text { correlation }\end{array}$ & ${ }^{1} \mathrm{H}-\mathrm{NMR}$ & $\begin{array}{c}{ }^{13} \mathrm{C}- \\
\text { NMR }\end{array}$ & ${ }^{1} \mathrm{H}-\mathrm{NMR}$ & $\begin{array}{l}{ }^{13} \mathrm{C}- \\
\mathrm{NMR}\end{array}$ & $\begin{array}{c}\text { HMBC } \\
\text { correlati } \\
\text { on }\end{array}$ & ${ }^{1} \mathrm{H}-\mathrm{NMR}$ & $\begin{array}{l}{ }^{13} \mathrm{C}- \\
\text { NMR }\end{array}$ \\
\hline 1 & $1.85(\mathrm{~m})$ & 37.39 & $\mathrm{C}-2$ & & 37.3 & $1.85(\mathrm{~m})$ & 37.39 & $\mathrm{C}-2$ & - & 37.3 \\
\hline 2 & $1.95(\mathrm{~m})$ & 32.02 & $\mathrm{C}-3$ & & 31.6 & $1.95(\mathrm{~m})$ & 32.05 & $\mathrm{C}-3$ & - & 31.6 \\
\hline 3 & $3.52(\mathrm{~m})$ & 71.93 & - & $3.52(\mathrm{~m})$ & 71.8 & $3.52(\mathrm{~m})$ & 71.93 & - & $3.52(\mathrm{~m})$ & 71.8 \\
\hline 4 & $\begin{array}{l}2.24(\mathrm{dd}, \\
J=1.44 ; \\
1.06) \text { and } \\
2.38, \mathrm{t})\end{array}$ & 42.42 & C-3,5,6 & & 42.3 & $\begin{array}{c}2.24(\mathrm{dd}, J= \\
1.44 ; 1.06) \\
\text { and } 2.38,1 \mathrm{H})\end{array}$ & 42.42 & C-3,5,6 & & 42.2 \\
\hline 5 & - & 140.87 & - & - & 140.8 & - & 140.87 & - & - & 140.8 \\
\hline 6 & $5.36(\mathrm{t})$ & 121.84 & $\mathrm{C}-8,10$ & $\begin{array}{c}5.357(\mathrm{br} \\
\mathrm{s})\end{array}$ & 121.7 & $5.36(\mathrm{t})$ & 121.85 & $\mathrm{C}-8,10$ & $\begin{array}{c}5.358(\mathrm{br} \\
\mathrm{s})\end{array}$ & 121.7 \\
\hline 7 & $1.99(\mathrm{~m})$ & 31.78 & C- $3,8,9$ & & 31.9 & $1.99(\mathrm{~m})$ & 31.78 & C- $3,8,9$ & - & 31.9 \\
\hline 8 & $2.00(\mathrm{~m})$ & 32.05 & C-5,6,9 & & 31.9 & $2.00(\mathrm{~m})$ & 32.05 & C-5,6,9 & - & 31.9 \\
\hline 9 & $0.94(\mathrm{~m})$ & 50.26 & C-7,8,12 & & 51.2 & $0.94(\mathrm{~m})$ & 50.28 & C- $7,8,12$ & - & 51.2 \\
\hline 10 & - & 36.64 & - & & 36.5 & - & 36.64 & - & - & 36.5 \\
\hline 11 & $1.02(\mathrm{~m})$, & 21.22 & C-5.8,9,13 & & 21.1 & $1.02(\mathrm{~m})$ & 21.22 & - & - & 21.1 \\
\hline 12 & $1.16(\mathrm{~m})$ & 39.82 & C-14,18 & & 39.8 & $1.16(\mathrm{~m})$ & 39.91 & C14,18 & - & 39.7 \\
\hline 13 & - & 42.35 & - & & 42.3 & - & 42.46 & - & - & 42.3 \\
\hline 14 & $1.00(\mathrm{~m})$ & 56.99 & $\begin{array}{c}\text { C-9,13,17, } \\
22\end{array}$ & & 56.8 & $1.00(\mathrm{~m})$ & 56.90 & $\begin{array}{c}\text { C-9,13, } \\
17,22\end{array}$ & - & 56.9 \\
\hline 15 & $\begin{array}{c}1.06(\mathrm{~m}) \\
\text { and } 1.58 \\
(\mathrm{~m})\end{array}$ & 24.45 & $\begin{array}{c}\mathrm{C}-8,9,14 \\
16\end{array}$ & & 24.3 & $\begin{array}{c}1.06(\mathrm{~m}) \text { and } \\
1.58(\mathrm{~m})\end{array}$ & 24.51 & $\begin{array}{c}\text { C-6,8, } \\
9,14\end{array}$ & - & 24.4 \\
\hline 16 & $\begin{array}{c}1.66(\mathrm{~m}) \\
\text { and } 1.25 \\
(\mathrm{~m})\end{array}$ & 29.07 & C- $18,20,22$ & & 28.3 & $1.09(\mathrm{~m})$ & 28.39 & C-17 & - & 28.4 \\
\hline 17 & $1.12(\mathrm{~m})$ & 56.08 & $\begin{array}{c}\text { C-8, 9,12, } \\
13,18 \\
\end{array}$ & & 56.0 & $1.12(\mathrm{~m})$ & 56.18 & $\begin{array}{l}\text { C-15,16, } \\
19,21,18\end{array}$ & - & 56.9 \\
\hline 18 & $0.85(\mathrm{~s})$ & 12.13 & C-8, 22 & $0.680(\mathrm{~s})$ & 11.0 & $0.85(\mathrm{~s})$ & 12.00 & $\mathrm{C}-8,22$ & $0.699(\mathrm{~s})$ & 11.9 \\
\hline 19 & $1,01(\mathrm{~s})$ & 19.54 & $\mathrm{C}-1,8,9,10$ & $1.01(\mathrm{~s})$ & 19.4 & $0.82(\mathrm{~s})$ & 19.18 & $\mathrm{C}-2,8$ & $1.01(\mathrm{~s})$ & 19.4 \\
\hline 20 & $1.16(\mathrm{~m})$ & 40.65 & $\begin{array}{c}\mathrm{C}-13,20,21, \\
23,24\end{array}$ & & 36.2 & $1,35(\mathrm{~m})$ & 36.30 & & & 36.2 \\
\hline 21 & $\begin{array}{c}1.03(\mathrm{~d}, \\
J=7.2 \mathrm{~Hz} \\
3 \mathrm{H})\end{array}$ & 21.23 & C-13,17 & $\begin{array}{c}1.02(\mathrm{~d}, \\
J=7.5 \mathrm{~Hz})\end{array}$ & 21.15 & $\begin{array}{c}0.92(\mathrm{~d}, \\
J=5.12 \mathrm{~Hz} \\
3 \mathrm{H})\end{array}$ & 18.92 & C-17 & $\begin{array}{c}0.92 \\
(\mathrm{~d}, J=6.4 \\
\mathrm{Hz}) \\
\end{array}$ & 18.8 \\
\hline 22 & $\begin{array}{c}5.00(\mathrm{dd}, J \\
=1.73 \mathrm{~Hz} \\
\text { and } 1.72 \\
\mathrm{~Hz})\end{array}$ & 138.46 & C- 20 & & 138.28 & $1,33(\mathrm{~m})$ & 34.07 & $\begin{array}{c}\mathrm{C}-23,24, \\
25,29\end{array}$ & & 33.9 \\
\hline 23 & $\begin{array}{c}5.15(\mathrm{dd}, \\
\mathrm{j}=1.75 \mathrm{~Hz} \\
\text { and } 1.73 \\
\mathrm{~Hz})\end{array}$ & 129.39 & C-24 & & 129.29 & $1.16(\mathrm{~m})$ & 26.20 & $\begin{array}{c}C-24,25 \\
28,29\end{array}$ & & 26.1 \\
\hline 24 & $1,55(\mathrm{~m})$ & 51.38 & C-22 & & 51.21 & $0.94(\mathrm{~m})$ & 45.96 & $\begin{array}{l}\text { C- } \\
20,21,22 \\
25,23,26\end{array}$ & & 45.9 \\
\hline 25 & $1.45(\mathrm{~m})$ & 32.03 & C-22 & & 31.88 & $1.66(\mathrm{~m})$ & 29.27 & $\begin{array}{l}\mathrm{C}-19, \\
23,24, \\
25,27, \\
28\end{array}$ & & 29.2 \\
\hline 26 & $\begin{array}{l}1.02(\mathrm{~d}, J \\
=13 \mathrm{~Hz})\end{array}$ & 21.21 & C-29 & & 21.06 & $\begin{array}{l}0.83(\mathrm{~d}, J=11 \\
\mathrm{Hz})\end{array}$ & 21.36 & $\begin{array}{l}\text { C-24, } \\
27,28 \\
29 \\
\end{array}$ & $0.83(\mathrm{t})$ & 19.8 \\
\hline 27 & 0.84 (br s) & 19.97 & C- 23,25 & $\begin{array}{l}0.795(\mathrm{~d} \\
J=6.5 \mathrm{~Hz})\end{array}$ & 19.79 & 0.84 (br s) & 19.13 & C-23,25 & $\begin{array}{l}0.814 \\
(\mathrm{~d}, J=6.5\end{array}$ & 19.3 \\
\hline
\end{tabular}


RASĀYAN J. Chem.

Vol. 13 | No. 4 |2552-2558| October - December | 2020

\begin{tabular}{|c|c|c|c|c|c|c|c|c|c|c|}
\hline & & & & & & & & & $\mathrm{Hz})$ & \\
\hline 28 & $1.16(\mathrm{~m})$ & 25.56 & C-26, 29 & $\begin{array}{l}0.846(\mathrm{~d}, \\
J=6.5 \mathrm{~Hz})\end{array}$ & 25.38 & $1,25(\mathrm{~m})$ & 23.20 & $\begin{array}{l}\text { C-22, } \\
24,25\end{array}$ & $\begin{array}{l}0.833 \\
(\mathrm{~d}, J=6.5 \\
\mathrm{Hz})\end{array}$ & 23.1 \\
\hline 29 & $0.81(\mathrm{t})$ & 12.41 & C- $25,27,28$ & $\begin{array}{l}0.845(\mathrm{t}, \\
J=7.5 \mathrm{~Hz})\end{array}$ & 12.22 & $0.85(\mathrm{t})$ & 12.19 & C-23,27 & $\begin{array}{l}0.845(\mathrm{t}, \\
J=7.5 \mathrm{~Hz})\end{array}$ & 12.2 \\
\hline
\end{tabular}

Compound 1 exhibits a weak antioxidant against DPPH radicals, however, $\beta$-sitosterol can protect against oxidative stress through modulation of antioxidant enzymes ${ }^{21}$ and Stigmasterol can decrease lipid peroxidation in the hepatic ${ }^{22}$. Also, both Stigmasterol and $\beta$-sitosterol are the main components of phytosteroids which will increase cholesterol excretion and reduce intestinal cholesterol absorption. ${ }^{23}$

\section{CONCLUSION}

Bioactivity-guided isolation of active compounds from the ethyl acetate fraction of B. macrocarpa wood bark extract gave compound (1). Structure elucidation based on spectral data suggested that compound (1) is a mixture of Stigmasterol and $\beta$-sitosterol. Both compounds are the first time isolated from $B$. macrocarpa (Tampoi).

\section{ACKNOWLEDGEMENT}

The authors are grateful to the IsDB project which has provided financial support with Grant Number: 137 / UN.17.11 / PL / 2019.

\section{REFERENCES}

1. R.M.A.P. Haegens, Blumea Suppement, 12(1), 1(2000)

2. T. Gunawan, Chikmawati, Sobir, Sulistijorini, Bioeksperimen Jurnal Penelitian Biologi, 2(2), 96(2016.)

3. N. K. Jauhari, D. Ibrahim, M. Ibrahim, M .N. A. Yahya, N. M. D. Nor, K. A. M. D. Isa, M. K. Ayob, M. N. Omar, N. Hazali, Sains Malaysiana, 42(2), 129(2013).

4. P. Milow, S. B. Malek, J. Edo, H. C. Ong, International Journal of Fruit Science, 14, 1(2014), DOI: $10.1080 / 15538362.2013 .801698$

5. Erwin, W.R. Pusparohmana, I.P. Sari, R. Hairani, Usman, Flo00Researc, 7(1977), 1(2019), DOI: $10.12688 /$ f1000research.16643.2

6. B.N. Meyer, N.R. Ferrigni, J.E. Putnam, L.B. Jacobsen, D.E. Nichols, J.L. McLaughlin, Planta Medica. 45(5), 31(1982), DOI:10.1055/s-2007-971236

7. Supomo, E. S. Syamsul, A. Apriliana, C. Saleh, Erwin, D. Lestari, Rasayan Journal of Chemistry, 12(3), 1340(2019), DOI: 10.31788/RJC.2019.1235264

8. Erwin, R.N. Nisa, Daniel, Indonesia Chimica Acta, 8(1), 52 (2015)

9. Erwin, Indonesia Chimica Acta, 8(2),11(2015)

10. J.A. O. Olugbuyiro, A. S. Banwo, A. O. Adeyemi, O. S. Taiwo, O. A. Akintokun, Rasayan Journal of Chemistry, 11(2), 798(2018), DOI:10.31788/RJC.2018.1121823

11. D.G.R. Aruan, T. Barus, G. Haro, P. Simanjunta, Rasayan Journal of Chemistry, 12(2), 947(2019), DOI: 10.31788/RJC.2019.1225204

12. M. O. Malpani, P. R. Rajput, K. V. Chinchole, S. S. Kapse, K. S. Ambarkar, Rasayan Journal of Chemistry, 12(4), 1901(2019), DOI: 10.31788/RJC.2019.1245447

13. D. Anokwah, A.Y. Mensah, I.K. Amponsah, E.A. Mireku, D.N. Mintah, Der Pharmacia Lettre, 8(20), 21(2016).

14. M. Borkataky, Der Pharmacia Littre, 7(5), 72(2015)

15. Bohari, A. Karolina, D.R. Pratiwi, Erwin, A. Rahmadi, EurAsian Journal of BioScience, 13(2), 2003(2019)

16. Erwin, S. Sulistyaningsih, I.W.Kusuma, International Journal of Pharma and BioSciences, 6(1), 598(2015)

17. U. U. Pateh, A. K. Haruna, M. Garba, I. Iliya, I.M. Sule, M.S. Abubakar, A.A. Ambi, Nigerian Journal of Pharmaceutical Sciences, 8 (1), 25 2009)

18. S. Subhadhirasakul1, P. Pechpongs, Songklanakarin Journal Science and Technology, 27 (Supplement 2), 561 (2005). 
RASĀYAN J. Chem.

Vol. 13 | No. 4 |2552-2558| October - December | 2020

19. L.K. Pierre, M.N. Moses, Journal of Innovations in Pharmaceuticals and Biological Sciences, 2(1), 96(2015).

20. T. Khammee, A. Rattanapittayapron, C. Rangjaroen, A. Jaratrungtawee, M. Kuno, Rasayan Journal of Chemistry, 12(4), 2273(2019), DOI: 10.31788/RJC.2019.1245412

21. M. Vivacons, J.J. Moreno, Free Radical Biology \& Medicine. 39, 97(2005) DOI: 10.1016/j.freeradbiomed.2005.02.025

22. S. Panda, M. Jafri, A. Kar, B.K. Meheta, Fitoterapia, 80(2), 123(2008), DOI: 10.1016/j.fitote.2008.12.002

23. S.B. Racette, X. Lin, M. Lefevre, C. A. Spearie, M. M. Most, L. Ma, R. E Ostlund, The American Journal of Clinical Nutrition, 91(1), 32(2010), DOI:10.3945/ajen.2009.28070

[RJC-5652/2020] 